\title{
Eu-doped $\mathrm{CaF}_{2}$ grown on $\mathrm{Si}(100)$ substrates by molecular beam epitaxy
}

\author{
X. M. Fang, T. Chatterjee, and P. J. McCann \\ School of Electrical Engineering and Laboratory for Electronic Properties of Materials, \\ University of Oklahoma, Norman, Oklahoma 73019 \\ W. K. Liu and M. B. Santos \\ Department of Physics and Astronomy and Laboratory for Electronic Properties of Materials, \\ University of Oklahoma, Norman, Oklahoma 73019 \\ W. Shan and J. J. Song \\ Department of Physics and Center for Laser Research, Oklahoma State University, Stillwater, \\ Oklahoma 74078
}

(Received 22 May 1995; accepted for publication 23 July 1995)

\begin{abstract}
$\mathrm{Eu}$ is incorporated into $\mathrm{CaF}_{2}$ films grown on $\mathrm{Si}(100)$ by molecular beam epitaxy using elemental Eu evaporation. Eu doping as high as 4.05 at. \% does not significantly degrade the surface morphology, indicating a relatively high solubility of $\mathrm{Eu}$ in $\mathrm{CaF}_{2}$. Photoluminescence spectra from Eu-doped $\mathrm{CaF}_{2}$ show strong blue emissions from $\mathrm{Eu}^{2+}$ ions in cubic sites. The inhomogeneous broadening of the zero-phonon line near $24190 \mathrm{~cm}^{-1}$ is reduced by $\sim 20 \%$ upon in situ annealing at $1100{ }^{\circ} \mathrm{C}$. (C) 1995 American Institute of Physics.
\end{abstract}

The increasing interest in the incorporation of rare-earth (RE) elements in epitaxial thin films of the alkaline-earth fluorides is motivated by potential applications in solid state microfilm or microcavity lasers. Growth of these materials on Si substrates offers potential compatibility with Si-based technology. Nd- and Er-doped $\mathrm{CaF}_{2}$ films grown on Si substrates by molecular beam epitaxy (MBE) have exhibited strong photoluminescence at $1.04 \mu \mathrm{m}$ and $1.54 \mu \mathrm{m}$, respectively. ${ }^{1-3}$ Eu-doped $\mathrm{CaF}_{2}$ bulk crystals have been extensively investigated ${ }^{4-9}$ for use as blue emission devices since a strong fluorescence at $420 \mathrm{~nm}$ is observed upon UV irradiation. Although laser action in Eu-doped $\mathrm{CaF}_{2}$ bulk crystals is hindered by excited state absorption, ${ }^{10}$ such films can be used as light emitters in multicolor thin-film electroluminescence devices. ${ }^{11}$ Recently, $\mathrm{Eu}^{2+}$ incorporated in $\mathrm{CaF}_{2}$ films grown on $\mathrm{Si}(111)$ substrates by $\mathrm{MBE}$ has been used as a sensitive probe of elastic strain in the heteroepitaxial layers. ${ }^{12,13}$ As reported, the growth of the Eu-doped $\mathrm{CaF}_{2}$ films was carried out by sublimation of a $\mathrm{CaF}_{2}$ single crystal containing $0.05 \%$ divalent Eu.

In this letter, we present the MBE growth of Eu-doped $\mathrm{CaF}_{2}$ on $\mathrm{Si}(100)$ substrates using elemental Eu evaporation. The incorporation of Eu in epitaxial $\mathrm{CaF}_{2}$ films was investigated by $\mathrm{x}$-ray photoelectron spectroscopy (XPS), reflection high-energy electron diffraction (RHEED), and photoluminescence (PL) measurements. We demonstrate that Eu concentrations as high as 4.05 at. \% do not significantly degrade the surface morphology, indicating a relatively high solubility of $\mathrm{Eu}$ in the $\mathrm{CaF}_{2}$ matrix. PL spectra from Eu-doped $\mathrm{CaF}_{2}$ show a strong blue to violet emission expected from $\mathrm{Eu}^{2+}$ ions in cubic sites. In situ post-growth annealing improves the morphology of the films and reduces the inhomogeneous broadening of the zero-phonon line of $\mathrm{Eu}^{2+}$ interconfiguration transitions.

Growth of Eu-doped $\mathrm{CaF}_{2}$ on $\mathrm{Si}(100)$ was carried out in

a)Electronic mail: fang@mailhost.ecn.uoknor.edu an Intevac Modular GEN II MBE system with a background pressure of $\sim 10^{-10}$ Torr throughout the deposition. The 3-inch diameter $p$-type $\operatorname{Si}(100)$ substrates (Silicon Sense, Inc.) were cleaned using the Shiraki method. ${ }^{14}$ The passivating oxide formed during the ex situ cleaning procedure was thermally desorbed in the growth chamber after several minutes at $\sim 1100{ }^{\circ} \mathrm{C}$. A surface prepared in this manner exhibits no discernible oxygen or carbon traces as examined by Auger electron spectroscopy. High purity polycrystalline $\mathrm{CaF}_{2}$ was evaporated from a graphite-coated PBN crucible at a typical growth rate of $20 \AA$ /min for a cell temperature of $1300{ }^{\circ} \mathrm{C}$. An elemental source of Eu was evaporated from a separate low-temperature effusion cell heated to $300-400{ }^{\circ} \mathrm{C}$ to give beam equivalent pressures of $3.5 \times 10^{-10}$ $1.9 \times 10^{-8}$ Torr.

$\mathrm{CaF}_{2}$ films grown on $\mathrm{Si}(100)$ are known to always exhibit a $\{111\}$ faceted morphology because the surface energy of the $\mathrm{CaF}_{2}(100)$ face is much larger than for $\mathrm{Si}(100)$ and $\mathrm{CaF}_{2}(111)$ faces. ${ }^{15}$ However, growth morphology and crystalline quality can be improved by in situ annealing. ${ }^{16,17}$ In our experiments, a $400 \AA \mathrm{CaF}_{2}$ buffer layer, a $3600 \AA \mathrm{Eu}-$ doped $\mathrm{CaF}_{2}$ layer, and a $200 \AA \mathrm{AaF}_{2}$ top layer were deposited on a $\mathrm{Si}(100)$ substrate at $580^{\circ} \mathrm{C}$. Most of the samples in this work were annealed in situ at $1100{ }^{\circ} \mathrm{C}$ for a few minutes at the end of growth.

XPS experiments were conducted at room temperature in an adjoining analysis chamber to eliminate complications caused by exposure to the atmosphere. This chamber is equipped with a VG100AX hemispherical analyzer and a twin-anode X-ray source used in conjunction with a VGX900 data acquisition system (Fisons Instruments). XPS spectra were taken using the $\mathrm{Al} K \alpha_{1,2}$ source $(h \nu=1486.6 \mathrm{eV})$ at a potential of $15 \mathrm{kV}$ and with $10 \mathrm{~mA}$ emission current. The binding energies were referenced to $\mathrm{F} 1 s$ at $684.5 \mathrm{eV}$. RHEED patterns were produced using a Varian electron gun operated at $9.5 \mathrm{keV}$ and at an angle of incidence of $1^{\circ}$ to $3^{\circ}$ and digitized using a CCD camera and data acquisition software developed by $k$-space Associates, Inc. Photolumines- 
Eu CELL TEMPERATURE $\left({ }^{\circ} \mathrm{C}\right)$

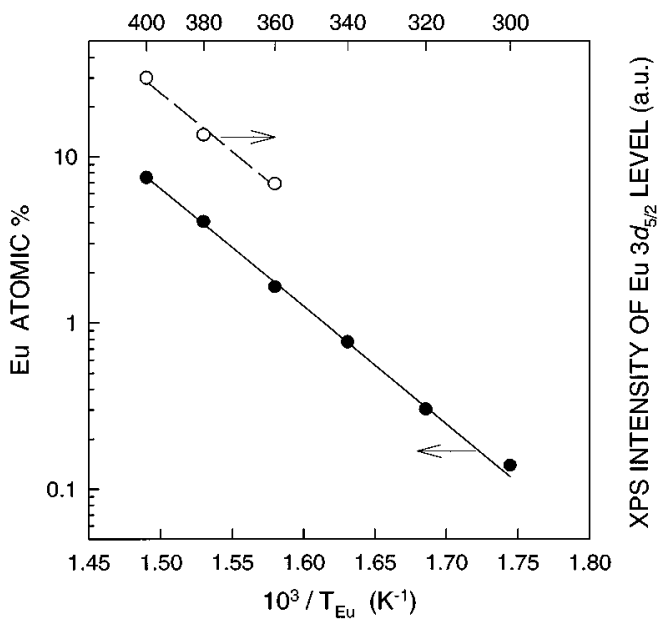

FIG. 1. Dependence of Eu concentration (solid circles) and integrated intensity of Eu $3 d_{5 / 2}$ XPS peak (open circles) on inverse Eu cell temperature.

cence (PL) measurements were performed at $10 \mathrm{~K}$ using the $325 \mathrm{~nm}$ line of a $\mathrm{HeCd}$ laser with an excitation power of 5-7 $\mathrm{mW}$. PL signals were collected by a $1 \mathrm{~m}$ Spex double-grating monochromator and detected by a S20 photomultiplier connected to a photon counting data acquisition system.

Figure 1 shows Eu concentrations in epitaxial $\mathrm{CaF}_{2}$ films grown at $580{ }^{\circ} \mathrm{C}$ as a function of inverse Eu cell temperature. The atomic percentage of Eu is calculated using the relations

$$
\text { at. } \%=\frac{J_{\mathrm{Eu}}}{J_{\mathrm{CaF}_{2}}+J_{\mathrm{Eu}}}=\frac{1}{\left(1+J_{\mathrm{CaF}_{2}} / J_{\mathrm{Eu}}\right)}
$$

and

$$
\frac{J_{\mathrm{CaF}_{2}}}{J_{\mathrm{Eu}}}=\frac{P_{\mathrm{CaF}_{2}}}{P_{\mathrm{Eu}}} \cdot \frac{\eta_{\mathrm{Eu}}}{\eta_{\mathrm{CaF}_{2}}} \sqrt{\frac{T_{\mathrm{CaF}_{2}} \cdot M_{\mathrm{Eu}}}{T_{\mathrm{Eu}} \cdot M_{\mathrm{CaF}_{2}}}},
$$

where $J$ is the beam flux, $P$ is the measured beam equivalent pressure, $\eta$ is the ionization efficiency relative to nitrogen (2.4 and 1.7 for Eu and $\mathrm{CaF}_{2}$, respectively), $T$ is the absolute temperature of the effusion cell, and $M$ is the molecular weight. Arrhenius behavior with an activation energy of 1.40 $\mathrm{eV}$ is observed up to $\sim 7.48$ at. $\%$.

The XPS spectra of Fig. 2 show Eu $3 d_{3 / 2}$ and Eu $3 d_{5 / 2}$ core levels from three Eu-doped $\mathrm{CaF}_{2}$ samples grown at different Eu cell temperatures. For comparison, the XPS spectrum of an undoped $\mathrm{CaF}_{2}$ film is also shown. The heights of the Eu $3 d$ peaks increase as the Eu cell temperature increases and the integrated intensities of these peaks depend exponentially on the inverse of the Eu cell temperature. As an example, the temperature dependence of the integrated intensity of the Eu $3 d_{5 / 2}$ peak is plotted in Fig. 1. The fit of the XPS data to an Arrhenius plot with an activation energy of $1.39 \mathrm{eV}$ indicates nearly the same temperature dependence as the Eu concentration.

Figure 3 shows RHEED patterns recorded with the electron beam along the [011] and [001] directions during growth of $\mathrm{a} \mathrm{CaF}_{2}$ film with a Eu concentration of $\sim 4.05$ at. \%. A well defined two-domain $(2 \times 1)$ RHEED pattern of the $\mathrm{Si}(100)$ substrate appears after oxide desorption [Fig.

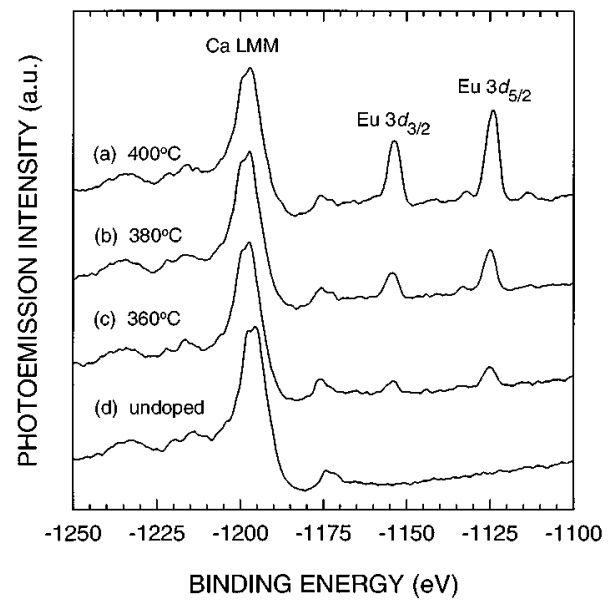

FIG. 2. XPS spectra showing Eu $3 d_{3 / 2}$ and Eu $3 d_{5 / 2}$ core levels and the X-ray induced Ca LMM Auger electron transition for samples grown using various Eu cell temperatures: (a) $400{ }^{\circ} \mathrm{C}$; (b) $380{ }^{\circ} \mathrm{C}$; (c) $360{ }^{\circ} \mathrm{C}$; (d) undoped.

3(a)]. The spotty RHEED patterns shown in Fig. 3(b), indicating three-dimensional island growth, were recorded after $400 \AA$ of $\mathrm{CaF}_{2}$ growth. The incorporation of Eu does not cause any apparent change in the surface structure of the $\mathrm{CaF}_{2}$ matrix as evidenced by comparing the RHEED patterns of the subsequent Eu-doped $\mathrm{CaF}_{2}$ layer [Fig. 3(c)] with those of the undoped $\mathrm{CaF}_{2}$ buffer layer [Fig. 3(b)]. However, surface degradation becomes visible under optical microscopy as the doping level reaches 7.48 at. \% even though the RHEED patterns remain unchanged. Precipitation of $\mathrm{Eu}$ at high doping levels may be responsible for the surface degradation. The effect of in situ annealing is evident in the RHEED patterns shown in Fig. 3(d). Sharp diffraction streaks with visible Kikuchi lines emerge immediately after the substrate temperature is raised to $1100{ }^{\circ} \mathrm{C}$, which indi-

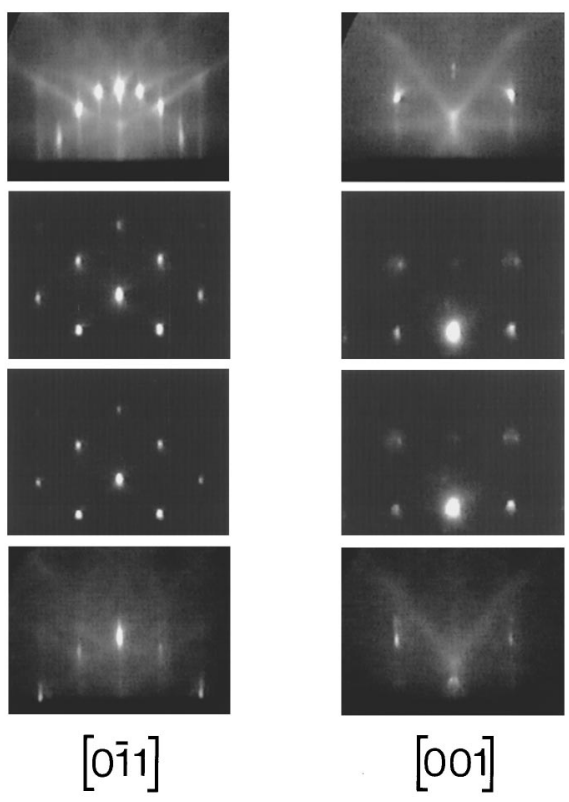

FIG. 3. RHEED patterns along the $[0 \overline{1} 1]$ and [001] azimuths recorded: (a) after desorption of oxide from Si substrate; (b) after deposition of a $400-\AA$ thick $\mathrm{CaF}_{2}$ layer; (c) after deposition of a $3600-\AA$-thick Eu-doped $\mathrm{CaF}_{2}$ layer; (d) after in situ annealing at $1100{ }^{\circ} \mathrm{C}$ for two minutes. 


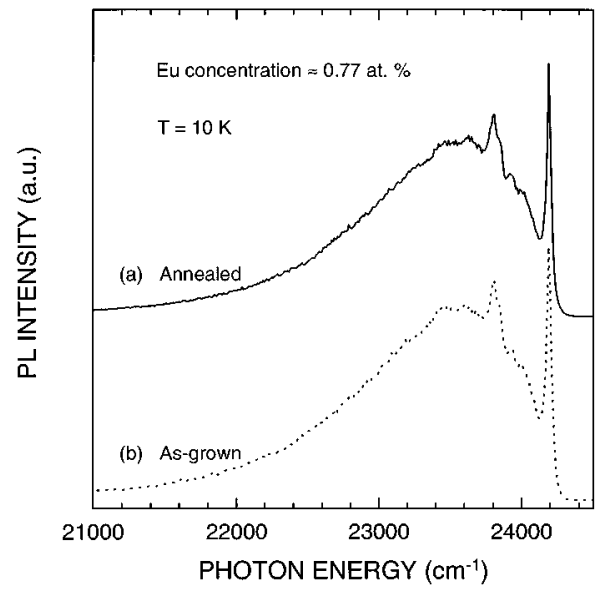

FIG. 4. PL spectra of (a) annealed at $\sim 1100{ }^{\circ} \mathrm{C}$ and (b) as-grown Eu-doped $\mathrm{CaF}_{2}$ films grown under the same conditions and with the same Eu concentration of $\sim 0.77$ at. \%. The full widths at half-maximum of the zero-phonon lines are $38 \mathrm{~cm}^{-1}$ and $46 \mathrm{~cm}^{-1}$, respectively. Both films are $\sim 4200 \AA$ A thick. Both spectra were taken at $10 \mathrm{~K}$.

cates a smooth surface. ${ }^{17}$ While in situ annealing improves the growth morphology by smoothing out the $\{111\}$ facets on the surface, ${ }^{17}$ it does not alleviate the morphological features attributed to Eu precipitation.

Figure 4(a) shows the PL spectrum of a $\mathrm{CaF}_{2}$ film with a $\mathrm{Eu}$ concentration of $\sim 0.77$ at. \%. A sharp zero-phonon line near $24190 \mathrm{~cm}^{-1}$ accompanies a broad vibronic sideband peaking at about $23500 \mathrm{~cm}^{-1}$. This spectrum is very similar to the fluorescence spectra of $\mathrm{Eu}^{2+}$ ions in $\mathrm{CaF}_{2}$ bulk crystals reported previously. ${ }^{5,8}$ The zero-phonon line shown in Fig. 4(a) arises from the electronic transition from the lowest lying $\Gamma_{8}\left(4 f^{6} 5 d\right)$ level of $\mathrm{Eu}^{2+}$ to the ${ }^{8} S_{7 / 2}\left(4 f^{7}\right)$ ground state. The peaks between the vibronic sideband maximum and the zero-phonon line are associated with the density of states maxima of various optical and acoustic phonons. All our samples, grown with various Eu concentrations, exhibit the spectral features shown in Fig. 4(a). No emission indicative of a ${ }^{5} D_{0} \rightarrow{ }^{7} F_{\mathrm{J}} \mathrm{Eu}^{3+}$ transition was observed in the entire scanned range of $15000-25000 \mathrm{~cm}^{-1}$.

Our PL data demonstrate the predominance of $\mathrm{Eu}^{2+}$ ions in cubic symmetry sites of $\mathrm{CaF}_{2}$ single crystal films prepared by MBE. This differs from previous reports on the preparation of Eu-doped $\mathrm{CaF}_{2}$ bulk crystals ${ }^{6,9}$ using the BridgmanStockbarger technique, where a small amount of Eu mixed with $\mathrm{CaF}_{2}$ in an excess of fluorine resulted in predominately $\mathrm{Eu}^{3+}$ ions in cubic or other lower symmetry sites. Chemical or irradiation reduction processes are required to convert the Eu ions in the bulk crystals from trivalent states to divalent states. Our results suggest that interstitial $\mathrm{F}^{-}$ions, substitutional $\mathrm{O}^{2-}$ ions, or other charged species which are necessary to compensate the additional positive charge of $\mathrm{Eu}^{3+}$ in $\mathrm{CaF}_{2}$ bulk crystals are absent in the MBE-grown Eu-doped $\mathrm{CaF}_{2}$ films. No excess $\mathrm{F}^{-}$ions are available because our experiments employ molecular $\mathrm{CaF}_{2}$ and elemental $\mathrm{Eu}$ beams provided by sublimation of $\mathrm{CaF}_{2}$ and $\mathrm{Eu}$ sources in a low background pressure environment. In Nd-doped $\mathrm{CaF}_{2}$ samples prepared by $\mathrm{MBE},{ }^{1} \mathrm{NdF}_{3}$ was intentionally selected to provide excess $\mathrm{F}^{-}$ions to enable substitution of $\mathrm{Nd}^{3+}$ ions for divalent cations in the $\mathrm{CaF}_{2}$ matrix. These results imply that the valence states of RE elements in fluorides can be controlled by using different RE dopant species.

To illustrate the effect of in situ annealing on the PL efficiency of $\mathrm{Eu}^{2+}$ ions in epitaxial $\mathrm{CaF}_{2}$ films, the PL spectra of annealed and as-grown films with the same growth conditions and Eu concentration are shown in Figs. 4(a) and 4(b), respectively. The peak intensity of the zero-phonon line remains nearly the same while the line width is narrowed by $\sim 20 \%$ and the integral intensity of the vibronic sideband is reduced by $\sim 13 \%$ upon annealing. It is evident that in situ annealing, which significantly improves the growth morphology, causes only minor changes in the PL efficiency of $\mathrm{Eu}^{2+}$. The insensitivity of the optical activity of $\mathrm{Eu}^{2+}$ to in situ annealing at temperatures as high as $1100{ }^{\circ} \mathrm{C}$ suggests that most of the Eu ions entering the $\mathrm{CaF}_{2}$ matrix are already located substitutionally in divalent cation sites during growth at $580{ }^{\circ} \mathrm{C}$. The reduced broadening of the zero-phonon line may result from embedded Eu ions moving closer to their equivalent positions upon annealing.

In summary, the growth of Eu-doped $\mathrm{CaF}_{2}$ thin films on $\mathrm{Si}(100)$ has been realized by MBE using an elemental $\mathrm{Eu}$ source and a substrate temperature of $580^{\circ} \mathrm{C}$. Good surface morphology is observed in samples with Eu concentration as high as 4.05 at. \%. Photoluminescence spectra from these samples show strong blue emissions attributed to $\mathrm{Eu}^{2+}$ ions in cubic sites. In situ annealing at $\sim 1100{ }^{\circ} \mathrm{C}$ reduces the inhomogeneous broadening of the zero-phonon line and significantly improves the growth morphology without dramatically changing the PL efficiency of $\mathrm{Eu}^{2+}$ ions.

We thank Joel Young for technical support with the MBE laboratory. This work was partially supported by the National Science Foundation through grant number OSR9108771.

${ }^{1}$ L. E. Bausá, R. Legros, and A. Muñoz-Yagüe, Appl. Phys. Lett. 59, 152 (1991).

${ }^{2}$ C. C. Cho, W. M. Duncan, T. H. Lin, and S. K. Fan, Appl. Phys. Lett. 61, 1757 (1992).

${ }^{3}$ E. Daran, R. Legros, A. Muñoz-Yagüe, C. Fontaine, and L. E. Bausá, J. Appl. Phys. 76, 270 (1994).

${ }^{4}$ A. A. Kaplyanskii and P. P. Feofilov, Opt. Spectrosk. (USSR) 13, 129 (1961).

${ }^{5}$ M. V. Hobden, Phys. Lett. 15, 10 (1965).

${ }^{6}$ J. L. Merz and P. S. Pershan, Phys. Rev. 161, 217 (1967).

${ }^{7}$ E. Loh, Phys. Rev. 184, 348 (1969).

${ }^{8}$ L. L. Chase, Phys. Rev. B 2, 2308 (1970).

${ }^{9}$ R. J. Hamers, J. R. Wietfeldt, and J. C. Wright, J. Chem. Phys. 77, 683 (1982).

${ }^{10}$ J. F. Owen, P. B. Dorain, and T. Kobayasi, J. Appl. Phys. 52, 1216 (1981).

${ }^{11}$ T. Suyama, K. Okamoto, and Y. Hamakawa, Appl. Phys. Lett. 41, 462 (1982).

${ }^{12}$ N. S. Sokolov, E. Virgilio, S. V. Gastev, S. V. Novikov, and N. L. Yakovlev, Sov. Phys. Solid State 31, 216 (1989).

${ }^{13}$ N. S. Sokolov, N. L. Yakovlev, and J. Almeida, Solid State Commun. 76, 883 (1990).

${ }^{14}$ A. Ishizaka and Y. Shiraki, J. Electrochem. Soc. 133, 666 (1986).

${ }^{15}$ L. J. Schowalter and R. W. Fathauer, CRC Crit. Rev. Solid State Mater. Sci. 15, 367 (1989).

${ }^{16}$ T. Asano, H. Ishiwara, and S. Furukawa, Jpn. J. Appl. Phys. 27, 1193 (1988).

${ }^{17}$ X. M. Fang, P. J. McCann, and W. K. Liu (unpublished). 